$\underline{\text { Title }}$

\title{
Mentalization-based treatment in clinical high-risk for psychosis: a rationale and clinical illustration
}

\author{
Authors \\ Martin Debbané ${ }^{1,2,3}$, Jallal Benmiloud², George Salaminios ${ }^{3}$, Alessandra Solida-Tozzi ${ }^{5}$, \\ Marco Armando2, Peter Fonagy 3,4 , Anthony Bateman 3,4
}

$\underline{\text { Affiliations }}$

${ }^{1}$ Developmental Clinical Psychology Research Unit, Faculty of Psychology and Educational Sciences, University of Geneva

2 Office Médico-Pédagogique Research Unit, Department of Psychiatry, University of Geneva School of Medicine, Switzerland

${ }^{3}$ Research Department of Clinical, Educational and Health Psychology, University College London, UK

${ }^{4}$ Anna Freud Centre, London, UK

${ }^{5}$ Department of Psychiatry, Service of General Psychiatry, Lausanne University Hospital (CHUV), Lausanne, Switzerland

\section{Corresponding Author:}

Martin Debbané

Adolescence Clinical Psychology Research Unit

Department of Psychology and Educational Sciences

University of Geneva

40 Boulevard du Pont d'Arve

1205 Geneva

Switzerland

Telephone: +41.22 .379 .94 .18$

Fax: +41.22.379.93.59

Email: martin.debbane@unige.ch

\begin{abstract}
:
Developmental clinical research in recent years has highlighted the value treating psychotic disorders at the earliest stage to reduce long-term morbidity. It is now suggested that treatment during the clinical high risk states (CHR), preceding by 1 to 4 years the onset of psychotic disorders, may delay or prevent the onset of psychosis, and contribute to a more positive prognosis. In this article, we wish to provide a rationale and clinical illustration of mentalization-based treatment (MBT) as an indicated preventive treatment for CHR. We will first review the notion of high-risk for psychosis, providing a trans-theoretical developmental framework for conceptualizing the clinical progression from sub-clinical towards clinical psychotic states. Second, we address the commonalities and differences between the constructs of mentalization and metacognition, and discuss their relevance in preventive psychotherapeutic treatment for CHR. Thirdly, we provide a clinical illustration of MBT to emerging psychosis. Finally, we conclude by discussing the specific contributions of MBT approach in youths at CHR, and the necessary research for evaluating its relevance in the context of risk for developing psychosis.
\end{abstract}


In the following, we consider mentalization-based treatment (MBT) as an indicated preventive treatment for individuals at clinical high-risk (CHR) for psychosis. Mentalization (thinking about thinking; (Fonagy 1991)) is a concept used both clinically and empirically to characterize a set of social cognitive processes that convey an understanding of human behavior as motivated by intentional mental states. We will first briefly review the developmental clinical milestones of emerging psychosis. The second section will specifically discuss the relevance of processes such as mentalization or metacognition during the premorbid and CHR phases of emerging psychosis. The last section will offer a clinical case illustration of mentalization-based treatment conducted with an adolescent female at CHR for psychosis. We conclude by highlighting how mentalization-based treatment may protect at-risk individuals from converting to clinical psychotic states.

\section{What is clinical high-risk for psychosis? A trans-theoretical developmental}

\section{framework}

Most theories seem to agree that a risk period precedes the onset of psychotic disorders, and that psychotic expression can be understood as a continuum, from non-clinical personality manifestations of schizotypy, to severe and chronic schizophrenic psychopathology (Figure 1) (Debbané 2015). In proposing a trans-theoretical model of developing psychosis, we first notice that all theories postulate a variant of what could be called a schizo seed, implying necessary but insufficient elements putting an individual at-risk for developing schizophrenia. Today, most consider this seed to be genetic in nature, with a number of gene constellations hypothesized to account for a significant proportion of variance in explaining psychosis (Arnedo et al. 2015). Second, all developmental theories identify an initial flip point, characterized by symptom expression that reaches, for the first time, the severity, frequency, and functional impact required by formal diagnostic criteria. This flip point is critical for a 
number of reasons: i) duration of untreated psychosis is related to severity of future morbidity (Hill et al. 2012); ii) the experience of psychotic episodes may themselves yield long-lasting harm on the individual (Emsley et al. 2013), and iii) collateral challenges incurred by hospitalizations, medication and significant interruptions in day-to-day functioning may play a role in prognosis.

Importantly, the CHR states precede this flip point, and potentially constitute an interval where preventive treatment is indicated. The two main contemporary approaches to CHR include the "basic symptoms" approach, which can be used in general population and helpseeking samples (Schimmelmann et al. 2015), and the "ultra-high risk" approach (Nelson et al. 2013) which is most often employed help-seeking samples. Basic symptoms are diagnosed on the basis of subjectively reported alterations in stress tolerance, affect, drive, thinking, speech, perception, motor action and central-vegetative functions (Schultze-Lutter et al. 2016). Basic symptoms can be distinguished from ultra-high risk because they seek to identify the earliest subtle disturbances predicting psychosis (Debbané et al. 2015), whereas ultra-high risk criteria targets imminent risk characterized by sub-clinical syndromes (attenuated psychotic symptoms (APS); brief limited intermittent psychotic symptoms (BLIPS)) encompassing unusual thought content, grandiose ideas, suspiciousness, perceptual abnormalities and disorganized communication (McGlashan et al. 2010).

Following the flip point, the clinical trajectories of diagnosed individuals roughly divide into three: 1) A first-episode of psychosis with no further psychotic breaks thereafter; 2) A firstepisode of psychosis followed by periods of remission and relapse; 3) A confirmation of a chronic diagnosis in the more severe range of expression, through gradual impairments and loss of flexible and adaptive functioning, likely to significantly handicap the autonomy and social function of the individual. 
Figure 1: A trans-theoretical model of developing psychotic disorders (adapted from Debbané, 2015)

----- Insert Figure 1 about here ------

It is estimated that the CHR period precedes the initial transition over a span from 1 to four years, and where individuals might already be seeking help for mental problems. CHR research therefore promotes an indicated prevention approach on individuals presenting the first signs of the emerging disorder (Schultze-Lutter et al. 2015).

\section{Mentalization, metacognition, and their relevance in CHR}

In this section, we will consider why psychotherapy focusing on specific processes such as mentalization or metacognition may be considered as potential indicated preventive method for individuals in the CHR stage. It has been noted elsewhere that in their psychotherapeutic usage, the terms mentalization and metacognition bear significant similarities as well as as a number of conceptual and clinical technique differences (Fonagy and Bateman 2016; Dimaggio et al. 2015). MBT constantly refers to a developmentally-informed understanding of mentalizing processes, both in its clinical and empirical applications (Fonagy and Luyten 2009), while Metacognitive Reflection and Insight Therapy (MERIT; (van Donkersgoed et al. 2014)) does not heavily rely on the developmental stages of metacognitive skills. Another difference, which stems from the theoretical heritage of both concepts, is the status of the body. In MBT, the notion of embodied mentalizing (Bateman and Fonagy 2012) refers to mental state activity which critically engages the person's bodily experience. The psychoanalytical background to the concept of mentalization further assumes that all mentalizing is elaborated on the basis of physiological arousal (Lecours and Bouchard 1997). While MBT does not rigidly adhere to Freudian metapsychology, it conserves the inclination to underscore the mutual dependency between mind and body. In MERIT, metacognitive activity is thought to assist in accurately 
representing body signals in order to integrate and regulate them, which translates a focus on appraisal mechanisms. Such subtle theoretical between differences MERIT and MBT might also yield different therapeutic techniques. While MERIT provides more clinical guidance to assess different levels of metacognitive functioning, MBT more readily assists the clinician in evaluating moment-to-moment assessment of mentalizing the therapeutic relationship, leading the clinician to focus more readily on the in vivo therapist-patient relationship experiences as material for learning from others (Bateman and Fonagy 2016; Fonagy and Allison 2014).

\subsection{Mentalization and metacognition as Higher-Order Cognition (HOC)}

The indisputable overlap between mentalization and metacognition (Fonagy and Bateman 2016) may be broadened to overlap with conceptual cousins such as theory of mind (Premack and Woodruff 1978), mindfulness (Bishop et al. 2004), reflective functioning (Katznelson 2014), cognitive and affective empathy (Shamay-Tsoory and Aharon-Peretz 2007), self and reality monitoring (Johnson et al. 1993), and other constructs devoted to capturing psychological processes at work when individuals think about mental states. The expression higher-order cognition (HOC; (Rudrauf 2014) may best capture the structural identity common to these hypothetical constructs, who all stem from hierarchical information processing networks of increasing complexity and abstraction, thought to critically assume a top-down, regulating influence on lower-level processing.

The common core of all HOC processes entails an awareness of self, and also an increased awareness of self-in-relation-to-other, and self-in-relation-to-the-world, which constitute the basic ingredients succeeding the developmental tasks of adolescents. Indirectly, HOC also echoes some phenomenological description of both the primary, basic sense of self, thought to rely on the brainstem (Solms 2013), and its elaboration through cortico-cortical connectivity which affords flexible adaptations to the specificities of the surrounding environment. 
However, the complexity of HOC may not be reducible to cerebral connectivity patterns; it is assumed that higher-order functioning occurs in a virtual workspace, where monitoring and regulation of information-processing patterns renders the system more efficient, and also more resilient in the case of organ injury or failure (normal aging, lesion, degeneration, etc.) (Rudrauf 2014). This brings us to the interesting idea that HOC processes, such as mentalization and metacognition, may not necessarily represent causal agents responsible for the onset of psychosis, but rather, resilience processes which may, in part, re-route functional connectivity during the pathological maturational patterns of the vulnerable brain in those at genetic and/or clinical risk for psychosis, and protect them from the full onset of psychosis. This is consistent with the hypothesis that if interventions strengthen HOCs, these could assist the individual in restructuring (re-routing) the functional organization of thinking towards less rigid, delusional and perseverative patterns of reality testing.

\subsection{Early HOC impairments in CHR youths: Towards an MBT approach to CHR}

The value of the HOC hypothesis is to underline the common architectural principles governing a number of different higher-order cognitive processes, such as mentalization and metacognition. On the basis of findings from behavioural, cognitive, neuroimaging and developmental research, Fonagy and Luyten (2009) have proposed to operationalize mentalization as multidimensional and organized along four separate but interrelated polarities: 1) automatic/controlled, 2) cognitive/affective, 3) internal/external and 4) self/other. Automatic mentalization is characterized by reflexive/intuitive, fast and unconscious processing of internal and external information, contrary to controlled mentalization, which is reflective, declarative, slow and conscious (Fonagy and Luyten 2009). The distinction between cognitive and affective mentalization refers to both the process and content of mentalizing activity. Accurate mental state inferences are based on the integration between cognitive (belief-desire reasoning and 
perspective-taking) abilities and emotional (affective empathy and mentalized affectivity thinking while feeling emotions) processes. At the same time the content of mentalizing activity of self and others may focus on belief-desire reasoning or affective material at varying degrees. The internal/external dimension refers to whether mental state inferences focus on mental interiors (thoughts, feelings) or externally perceived features (physical, visual) of the self or others. Finally, the self/other dimension refers to the commonality between the developmental and brain systems underpinning the attribution of mental states of the self and of others (Murray et al. 2015). Accurate mentalizing inferences are primarily dependent on the relative balance between the systems underpinning each dimension, while biased inferences associated with psychopathology emerge under conditions that lead to a loss of balance (Luyten and Fonagy 2015). For example, hyper-reflexivity in psychotic patients might reflect a polarization on the internal-cognitive polarities of mentalization, effectively splitting off from the externalaffective dimensions of experience.

We have reviewed elsewhere the evidence suggesting that impairments in the mentalizing dimensions precede the onset of frank psychosis (Debbané et al. under review). Studies examining Theory of Mind (ToM) report subtle impairments in the Cognitive/Other polarities of mentalization in youths presenting with sub-clinical signs of psychosis (Clemmensen et al. (2015), and more importantly, that robust ToM capacities appear to reduce the tenacity of psychotic-like experiences in youths (Bartels-Velthuis et al. 2011). Together, these studies and others highlight a number of different HOC impairments experienced by at-risk individuals before the onset of psychosis.

From the standpoint of MBT, impairments in HOCs in these youths should be analyzed in relation to the types of interpersonal contexts in which they are more likely to emerge (Fonagy and Luyten 2009; Mayes 2000). Brent \& Fonagy already highlighted how significant social distress experienced in early attachment relationships, and within the adolescent's interpersonal 
context, ignites significant arousal that is likely to disturb the integration of mentalization dimensions sustaining the interpretation of self and other behaviors (Brent and Fonagy 2014). As psychosis most frequently presents itself in late adolescence or early adulthood, amidst profound socio-relational changes in relation to developmental tasks, we argue that aiming to strengthen the robustness of mentalization using both individual and family MBT (Rossouw and Fonagy 2012) may significantly reduce the intensity of CHR attenuated psychotic symptoms, as well as harmonize the socio-functional integration by fostering social cognitive understanding of self and others. In the following section, we will illustrate the treatment rationale and method that support the clinician in focusing on mentalization in a psychotherapy, through the case presentation of an adolescent female diagnosed at CHR for developing psychosis.

\section{A clinical illustration from an MBT standpoint}

The following clinical illustration presents a psychotherapeutic treatment provided to a patient with $\mathrm{CHR}$, where MBT treatment modalities were modeled to those proposed to adolescents with emerging borderline personality disorders (Rossouw and Fonagy 2012). The psychotherapist is trained in psychodynamic psychotherapy, and has additionally been trained in MBT at the Anna Freud Center in London, and follows regular MBT supervision in her clinical setting in Geneva, Switzerland. The patient follows a weekly individual session, and a monthly family session.

The outpatient treatment was initiated after referral from the pediatric department at the University Hospitals in Geneva. The evaluation took place over several appointments, and psycho-education for MBT was provided in the context of the first family sessions. After the evaluation was complete, a mentalizing case formulation was written jointly with the patient and revised with the patient's mother before psychotherapy began: 


\section{Box.1 Case Formulation of Khim}

\section{Personal History}

Khim is a 16-year-old adolescent girl, with one prior hospitalization for a depressive disorder with psychotic features at age 15. Khim's parents, originally from Asia, met each other before they came to Switzerland. Each parent's life is marked by a history of trauma, professional hardships, multiple familial separations and relationship discontinuities. Father was emotionally unstable, and left mother for another woman; he subsequently had multiple short-term relationships. Mother describes herself as impulsive and distrustful of others. The parents' separation occurred when mother was pregnant with Khim; early, the child was exposed to violent disputes in the context of her parents' on and off relationship, and starting age 5 she was brought up by her mother and had no contact with her father until early adolescence.

Between 2 to 4 years of age, Khim received counseling for important delays in language acquisition, attention and emotional regulation, as well as feeding and sleeping problems. Her attachment to her mother was assessed as "disorganized". Her relationship style with other children in kindergarten, and then in preschool, was described as rude, authoritarian and sometimes not respecting personal boundaries. Khim also suffered from intense separation anxiety, haunted by the idea that one day her mother will never come back to pick her up from school.

During high-school, Khim was described as a smart student, although often daydreaming, quite inattentive but also occasionally engaging in desperate attempts to draw attention from her peers. Regular misunderstandings with her peers made her experience many rejections. Furthermore, Khim felt torn apart between teachers that she appreciated, but with whom she 
could have rows, and her mother's critical comments about them, leaving her feeling very depressed.

\section{Engagement in therapy}

Khim consulted with a number of therapists in her life, whom she says have helped her to feel better, yet she also feels that problems in her life seem to persist. Her motivation for therapy was high but marked by the ambivalent belief that nobody could really help her out. More specifically, she regularly felt misunderstood, and this could lead her to experience selfdisorganization, characterized by convoluted explanations of reality that plunged her into states of confusion and sometimes dissociation. Feeling misunderstood could also make her shut down and engage in paranoid thinking about the malevolent intentions of others. In a face-toface setting, she interpreted silences as very distressful, where she experienced herself as disappearing from the therapist's mind. We agreed her tendency to over-attribute intentions to the therapist (hypermentalizing style) should be monitored when it arose during sessions; it was proposed that the therapist may inadvertently promote hypermentalizing through bouts of silence, or by not making her thought processes explicit to Khim, and that this should be worked out during the session.

\section{Relationship difficulties}

Khim and her mother regularly engage in conflict. Khim experiences intense social anxiety when she feels misunderstood or uninteresting. Interpersonal conflict, whether or not it directly involves her, will trigger a sense of being in danger or a need to withdraw. She regularly thinks that her friends could be plotting against her behind her back, revealing her secrets to everybody, or planning to humiliate her publicly. Her beliefs concerning the thoughts of others are often rigidly embedded in certainty; she often fails to appreciate the opacity and the changing nature of her mind and the mind of others.

\section{Psychosis}


Schizotypal phenomena were present at a very early age. For example, Khim vividly remembers entire periods of her childhood where she was convinced of living the life of this television idol, feeling the notice of her "fans" on the bus, but also noting that transitions back to her normal identity were accompanied by a sense of loosing her ordinary self. She further remembers a persistent and intense fear of potentially vomiting internal organs out of her body. More recently, she reports often looking at herself in the mirror for an hour, attempting to " $g e t$ back into my identity by touching my face and making mimics". She describes experiencing important distress when, feeling under stress, she can start hearing mocking voices. These are relatively rare and she understands the voices as "my internal voice taking on another person's voice to criticize me". She is worried that one day she might become crazy, preventing her from finding a loving boyfriend to create her own family.

We administered the Structured Interview for Prodromal Syndromes (SIPS; (McGlashan et al. 2010)) when she was 15 years old. At the time, her CHR symptoms qualified for the Attenuated positive symptoms prodromal syndrome (APS) on the basis of Unusual thought content/Delusional ideas (P1), Suspisciousness/Persecutory ideas (P2), and Perceptual abnormalities/Hallucinations (P4).

\section{Mentalizing}

\section{Concrete mentalizing (Teleological mode)}

When Khim feels unsettled about the psychological availability of a close other, her increasing distress can lead her to seek clues about mental states in physical reality. For example, she will interpret her mother's mood on the basis of physical indices, like the way her mother disposes the dishes in the kitchen sink; if her mother stacks dishes in the left sink, then she must be disappointed with Khim. Physical reality becomes the ultimate testimony of her mother's internal state.

Inside-Out Mentalizing (Psychic Equivalence) 
In close relationships, Khim has the tendency to become very suspicious and jealous. Doubting her friends' loyalty can rapidly escalate into certainty about their disloyal mental states: what she thinks inside becomes real outside, in her friends' minds and intentions.

\section{Hyper-reflexive Mentalizing (Pretend Mode)}

Khim can become quite entangled with her introspective thoughts. During instances where she feels misunderstood by friends for example, she appears to retreat in forms of hyperreflexive self-evaluation. She describes this experience as beginning with the uncertainty about how others view her. She feels herself confusingly trying to conform to how she believes others perceive her, even though she has no clear picture of how this should be. She reports a sense of disconnection to herself in such instances, and at times, she experienced auditory hallucinations with hostile voices commenting on her behavior.

Before illustrating the MBT treatment undertaken with Khim through two clinical vignettes, we will briefly summarize the main technical points common to MBT applications to different clinical populations (Bateman and Fonagy 2012); the reader can refer to other works for the most recent practical guides detailing the MBT practice (Bateman and Fonagy 2016; Debbané 2016). Four main clinical foci guide the MBT therapists in their practice: 1) the therapeutic stance; 2) a focus on affect; 3) generating a process of mentalizing in therapy; 4) Using the therapist-patient relationship to generate affective learning in session.

The therapeutic stance in MBT reunites a number of different elements at the basis of good clinical practice and sustaining a working alliance with the patient. These include the therapist's warmth, curiosity about mental states, and her ability to foster a process of joint attention commonly examining the patient's mental states and mental states governing others' behavior. Critically, the therapeutic stance in MBT assumes that mental states are opaque in nature, and 
therefore knowledge about mental states of self and others cannot be held with certainty. This point is crucial in psychotherapy with psychotic patients who can exhibit rigid certainty about mental states of others. Here, the therapist may employ psycho-educative techniques to mark opacity as an essential feature of mental state knowledge.

The MBT clinician always seeks to make affect a topic of joint attention during the session, and with individuals with compromised development of mentalization skills (as can be the case of high-risk for psychosis youths), affective states can coalesce with unsettling perceptive and bodily aberrations, together subjecting the patient to distressing self-experiences. In MBT for psychosis, we would emphasize that embodied mentalizing represents a particular area of investigation for the clinician. More specifically, the mentalizing process may be sustained by attention to and description of the "internal states" the patient experiences during aberrant perceptive and bodily experience, where the therapist would help to disentangle these from thoughts about mental states of self and others which tend to aggregate and confuse these patients. The generation of the mentalizing process is key to the third clinical foci, and guides the activity and rhythm of interventions of the clinician practicing MBT. The clinician is active(inquisitive stance) whilst respecting the patient's availability to engage mentalizing. The therapist will systematically monitor ruptures in the patient's or her own mentalizing process, and prioritize the rekindling of the mentalizing process. In circumstances where affect about the therapeutic relationship is hindering the mentalizing process, the clinician practicing MBT will not hesitate to bring this relationship at the center of focus of joint attention. As further illustrated in the second clinical vignette, this psychodynamic technique offers the opportunity for in vivo experiencing and learning about the interpersonal consequences mental states can bear on self and others, and the utility of explicitly exploring these in an open and safe dialogue. 
Vignette 1a: Working with concrete mentalization in CHR youths.

This interaction takes place at the beginning of a session. As Khim enters the consultation room, the therapist notices that she appears to be disturbed by something, as she is scanning the whole room more than she normally does. The session starts after Khim and the therapist settle in their chairs :

Therapist : How are you today Khim?

Khim: I am doing ok. (speech not concordant with being ok)

Therapist: Hmmm... what kind of "ok" is it today then?

Khim: Don't know... just ok I guess.

Therapist: (seeing a potential impasse, and making her thoughts available to the patient) I guess I am asking the question because I am noticing you were looking all around the consulting room, a bit more than usual it seems to me, and I wondered what you were looking at, or what was on your mind as you were looking around? (modelling the opacity of mental states)

Khim: No... There is nothing (silence)...

Therapist: Ok...

Khim: It is just that... (silence). Well ... I guess... this is kind of boring to you.

Therapist: Oh, really? Hmmm... let's see... I don't really feel bored right now, but you might be picking up something I am not aware of... (modelling opacity of self mental states). Tell me, is there anything that I am doing suggesting that I am bored? ("not knowing stance" and “curiosity”)

Khim: Hum....(pause). When I entered, you did not say a word.... And there were the chairs too.... 
Therapist: The chairs? What about the chairs?

Khim: You've spaced them out... you're distant. (Concrete mentalizing)

In this dialogue, the therapist sets her therapeutic stance, by modelling the opacity of her own mind and the mind of the patient, and striving to maintain a not-knowing attitude, leaving things open to discussion. She also takes into consideration the patient's belief, and tries to answer as authentically as possible by engaging in calibrated self-disclosure about how bored she feels. Importantly, she intervenes to clarify elements that seemed surprising or odd, like the concrete mentalizing of the distance between the chairs, which seemed to mark a breakdown in mentalizing.

At this point, Khim started to divert her attention from the exchange with the therapist, and to look elsewhere as she went into a kind of automatic mode. She related factual and rather superficial discourse about school and class workload, in a prototypical patient-to-therapist discourse. The emotional climate had suddenly changed, from a sense of strangeness to a sense of emotionally detached, day-to-day talk (pretend mode). The therapist had a hard time making sense of this switch, and could not follow Khim's storyline:

Vignette 1b: Working with concrete mentalization (continued).

Therapist: I am really sorry to interrupt you Khim, but in my mind right now (making the therapist's thought processes explicit), I am still struggling to understand what you meant a few minutes ago when you mentioned "the distance between the chairs". Do you mind if we go back to this? (Stop and rewind).

Khim: ... (does not answer but appears to become still and a bit stiff) 
Therapist: If I remember what you said, it sounded like you saw the chairs apart, and also you sensed I was distant? Did I get that right?

Khim: Yes, that is what I said. From the start I knew you were bored, that is why you put the chairs apart.

Therapist: Hmm I didn't realize that... now that I'm thinking about it, I can imagine that coming here and having the feeling I'm distant for some reason must be kind of unpleasant?

Khim: I don't like the silence here.

Therapist: Hmm.. what about the silence?

Khim: I don't like it. It's kind of useless to talk today.

Therapist: So let me see if I got this right. We started off and I was silent, and that might be linked to your feeling that I am bored today and perhaps distant, like the chairs? I can understand you feel its useless to talk to someone distant, I think I would feel the same way. (empathic validation)

Khim : Yes, I don't like arriving somewhere and people being silent.

Therapist: How does that work for you, does it make you feel anything unpleasant

Khim : Like I am disturbing people... like they don't care, they are not interested in what I have to say.

Therapist: Is that how you felt here, a moment ago?

Khim : Yeah kind of ... but it's ok.

Therapist: You know Khim I had no idea this silence made you feel you were disturbing me; thanks for telling me, I will watch out for this from now, because I don't want my silences, or 
other things I may say or not say, to generate those feelings. These feelings seem to make you feel that it is not worth talking with me, right?

Khim : I think that is about right.

Therapist: Could you also let me know if you notice thoughts and feelings emerging during our sessions? That would help me, because I can't read your mind, so telling me what is on your mind will also help me understand you better. (psycho-educating on the opacity of minds, and how making them more explicit can help us better understand each other). Tell me then, now that we are talking about it, do I appear as tired and distant to you?

Khim : I'm not so sure anymore. The feeling is kinda gone... I just remembered there was something I really wanted to tell you about my friend, you know the one who's parents just got divorced?

The use of the therapeutic stance helped the therapist to work with the concrete mentalizing operating for Khim at the beginning of the session, and the a focus on the mentalizing process helped them both to disentangle the physical, perceptual reality from its concrete association to mental states, and its consequence on feelings in the interaction with the therapist. In the second vignette, we illustrate further how the mentalizing process may directly implication the therapist-patient interaction within the session to provide content for learning about interpersonal relationships:

Vignette 2: Working with attenuated psychotic symptoms (ideas of reference as an example) 
Khim and her therapist are discussing how distressed she felt coming to the session. It is unclear to Khim why she feels this way; she mentions that some ideas of reference emerged during the bus ride to the session, where she felt she could read the minds of the people in the bus :

Khim : It was just really uncomfortable. I had to stop looking at them.

Therapist : Did you feel everybody in the bus thinking things about you?

Khim : Pretty much, it got more intense if I looked at them.

Therapist : My goodness, that must be an awful feeling... you still seem quite agitated about it (Khim nodding...); I'm just wondering, am I creating that same feeling in you, that I am thinking things about you?

Khim : (low voice) ... yes.

Therapist : And are you reading my thoughts now?

Khim : yes.

Therapist : Hmmm... I'm a bit stuck here, because I can tell that what you are experiencing is related to you reading my thoughts, but at the same time, if I tell you I am thinking something different than what you perceive, then you might think I a lying to you about my thoughts. Am I getting this part right?

Khim : (slightly relieved)... yes.

Therapist : So what are we to do? Could we try something out where I note my thoughts on a piece of paper, and then you tell me what you think I wrote?

Khim : Sure.

Therapist : Ok, so you told me that in the bus, you felt reading the minds of others when you looked at them, right? (Khim nods). So here, what we could try is to look at each other in the 
eye, really briefly, and then I will write down the thought that passed through my mind, and you then tell me what it was you read, ok?

Khim : Ok.

Therapist : (... looks at Khim and then writes thought). So what was it that you read in my mind ?

Khim : Not sure I can say it....

Therapist : Do you want to give it a try?

Khim : You think I'm crazy.

Therapist : Hmmm... well, I don't remember ever thinking that, but like I mentioned, I am not sure you believe me when I say that.

Khim : You are right, I don't really believe you.

Therapist : That is what I thought, so again I am stuck here, because I feel whatever I say, you can legitimately question it, right?

Khim : (supporting a bit more eye contact) Yes.

Therapist : Ok, so I suggest we still have a look at what I wrote, what do you say...?

The therapist repeated the exercise a few times, and prompted Khim to evaluate how certain she was about the contents of the therapist's mind. She evolved from a $9 / 10$ to a $4 / 10$, which surprised her. The technique used by the therapist was not pre-defined, but rather used on the spot to employ the in vivo experiences within the therapist-patient interaction the foster selfother differentiation, whist at the same time validating Khim's experience and being able to contain it emotionally during the session. Importantly, the therapist made sure to validate Khim's suspiciousness about the contents of her mind, and constantly checked back whether 
she has a good understanding of how she was feeling within the exercise. Moreover, the therapist wanted to make sure not to scare or be intrusive with the proposition to examine mindreading in the session, by checking and respecting Khim's experience at every step. Conversely, the therapist wanted to avoid arguing about who was right about the thoughts themselves, but rather to model how "mind-reading" made each other feel in the context of their current session. This lead Khim to notice that the openness of the therapist's mind made her feel less suspicious and more engaged in the session.

\section{Conclusion}

The main objective of this paper was to offer a rationale for mentalization-based treatment (MBT) as an indicated preventive treatment option for CHR states for developing psychosis, and to illustrate the treatment approach through clinical vignettes. Because MBT focuses on a key set of social cognitive processes that sustains social functioning, and because it integrates interpersonal relationships, we feel it responds to the needs identified by previous research for preventive treatment to foster resilience and assist individuals in managing the socio-relational challenges they feel cause the most important distress at this stage of their development (Rapado-Castro et al. 2015).

We recently asked Khim to reflect on her process of change during therapy. She first responded, with apparent emotion, that «things inside me feel so much more connected ». She then described that in the past, she felt other people possessed the capacity to understand each other, a capacity which she lacked. She experienced the interpersonal world as though in a mist that engendered a lot of confusion and anxiety. She now feels this mist has dissipated, and she approaches relationships with more confidence. When asked more specifically about the interventions she thought were helpful, she underlined the psycho-educative approach of systematically noticing the things that activated her emotions, and linked them to her thought 
processes helped her, and that globally the whole approach was « very transparent » and made clearer how to deal with medication issues, and also how to accept the risk that her symptoms were indicating whilst being able to live with it. Globally, she felt reconnected with a sense of hope and empowered through « a capacity to care for myself ».

MBT is certainly not the sole therapy that could be indicated for CHR, and through the concept of $\mathrm{HOC}$, we argue that psychotherapeutic actions focusing on mentalization or metacognition are likely to afford flexibility for individuals to restructure (re-route) their functional organization of thinking towards less rigid, delusional and perseverative patterns of reality testing, whilst they face real neurogenetic and/or multiple other risk for the development of psychosis.

\section{Compliance with Ethical standards}

The patient described in this manuscript gave her full consent after reading the excerpts of text relating to her treatment.

Funding: This work was funded by a Swiss National Science Foundation Grant (100019159440) awarded to M.D.

Conflict of interest: None of the authors declare any conflict of interest.

Acknowledgements: We would like to thank Deborah Badoud, Yasmina Lakeshaft Lachat, and Larisa Morosan for their help on the manuscript.

\section{References}

Arnedo, J., Svrakic, D. M., Del Val, C., Romero-Zaliz, R., Hernandez-Cuervo, H., Molecular Genetics of Schizophrenia, C., et al. (2015). Uncovering the hidden risk architecture of the schizophrenias: confirmation in three independent genome-wide association studies. Am J Psychiatry, 172(2), 139-153. 
Bartels-Velthuis, A. A., Blijd-Hoogewys, E. M., \& van Os, J. (2011). Better theory-of-mind skills in children hearing voices mitigate the risk of secondary delusion formation. Acta Psychiatr Scand, 124(3), 193-197.

Bateman, A. W., \& Fonagy, P. (2012). Handbook of Mentalizing in Mental Health Practice. Arlington: American Psychiatric Publishing.

Bateman, A. W., \& Fonagy, P. (2016). Mentalization-Based Treatment for Personality Disorders: A Practical Guide. Oxford: Oxford University Press.

Bishop, S. R., Lau, M., Shapiro, S., Carlson, L., Anderson, N. D., Carmody, J., et al. (2004). Mindfulness: A proposed operational definition. Clinical Psychology-Science and Practice, 11(3), 230-241.

Brent, B., \& Fonagy, P. (2014). A Mentalization-Based Treatment Approach to Disturbances of Social Understanding in Schizophrenia. In P. H. Lysaker, Dimaggio, G., Brüne, M. (Ed.), Social Cognition and metacognition in Schizophrenia (pp. 245-257). Sam Diego: Elsevier.

Clemmensen, L., van Os, J., Drukker, M., Munkholm, A., Rimvall, M. K., Vaever, M., et al. (2015). Psychotic experiences and hyper-theory-of-mind in preadolescence - a birth cohort study. Psychol Med, 1-15.

Debbané, M. (2015). Schizotypy: a developmental perspective. In O. Mason, \& G. Claridge (Eds.), Schizotypy: New Dimensions: Routledge.

Debbané, M. (2016). Mentaliser. Louvain-la-Neuve, Belgium: De Boeck Supérieur.

Debbané, M., Eliez, S., Badoud, D., Conus, P., Fluckiger, R., \& Schultze-Lutter, F. (2015). Developing psychosis and its risk States through the lens of schizotypy. Schizophr Bull, 41 Suppl 2, S396-407. 
Debbané, M., Salaminios, G., Badoud, D., Luyten, P., Solida-Tozzi, A., Fonagy, P., et al. (under review). Attachment, neurobiology, and mentalization along the psychosis continuum.

Dimaggio, G., Montano, A., Popolo, R., \& Salvatore, G. (2015). Metacognitive Interpersonal Therapy for Personality Disorders: A Treatment Manual. London: Routledge.

Emsley, R., Chiliza, B., Asmal, L., \& Harvey, B. H. (2013). The nature of relapse in schizophrenia. BMC Psychiatry, 13, 50.

Fonagy, P. (1991). Thinking about thinking: some clinical and theoretical considerations in the treatment of a borderline patient. [Case Reports]. Int J Psychoanal, 72 ( Pt 4), 639656.

Fonagy, P., \& Allison, E. (2014). The role of mentalizing and epistemic trust in the therapeutic relationship. Psychotherapy (Chic), 51(3), 372-380.

Fonagy, P., \& Bateman, A. W. (2016). Adversity, attachment, and mentalizing. Comprehensive Psychiatry, 64, 59-66.

Fonagy, P., \& Luyten, P. (2009). A developmental, mentalization-based approach to the understanding and treatment of borderline personality disorder. Dev Psychopathol, 21(4), 1355-1381.

Hill, M., Crumlish, N., Clarke, M., Whitty, P., Owens, E., Renwick, L., et al. (2012). Prospective relationship of duration of untreated psychosis to psychopathology and functional outcome over 12 years. Schizophr Res, 141(2-3), 215-221.

Johnson, M. K., Hashtroudi, S., \& Lindsay, D. S. (1993). Source monitoring. Psychological Bulletin, 114(1), 3-28.

Katznelson, H. (2014). Reflective functioning: a review. Clin Psychol Rev, 34(2), 107-117. Lecours, S., \& Bouchard, M. A. (1997). Dimensions of mentalisation: Outlining levels of psychic transformation. International Journal of Psycho-Analysis, 78, 855-875. 
Luyten, P., \& Fonagy, P. (2015). The neurobiology of mentalizing. Personal Disord, 6(4), 366-379.

Mayes, L. C. (2000). A developmental perspective on the regulation of arousal states. Seminars in Perinatology, 24(4), 267-279.

McGlashan, T. H., Walsh, B. C., \& Woods, S. W. (2010). The psychosis-risk prodrome: handbook for diagnosis and follow-up. New York: Oxford University Press.

Murray, R. J., Debbane, M., Fox, P. T., Bzdok, D., \& Eickhoff, S. B. (2015). Functional connectivity mapping of regions associated with self- and other-processing. Hum Brain Mapp, 36(4), 1304-1324.

Nelson, B., Yuen, H. P., Wood, S. J., Lin, A., Spiliotacopoulos, D., Bruxner, A., et al. (2013). Long-term follow-up of a group at ultra high risk ("prodromal") for psychosis: the PACE 400 study. [Evaluation Studies

Research Support, Non-U.S. Gov't]. JAMA Psychiatry, 70(8), 793-802.

Premack, D., \& Woodruff, G. (1978). Does the Chimpanzee Have a Theory of Mind. Behavioral and Brain Sciences, 1(4), 515-526.

Rapado-Castro, M., McGorry, P. D., Yung, A., Calvo, A., \& Nelson, B. (2015). Sources of clinical distress in young people at ultra high risk of psychosis. Schizophr Res, 165(1), $15-21$.

Rossouw, T. I., \& Fonagy, P. (2012). Mentalization-based treatment for self-harm in adolescents: a randomized controlled trial. J Am Acad Child Adolesc Psychiatry, 51(12), 1304-1313 e1303.

Rudrauf (2014). Structure-Function Relationships behind the Phenomenon of Cognitive Resilience in Neurology: Insights for Neuroscience and Medicine. Advances in Neuroscience, 4, 1-28. 
Schimmelmann, B. G., Michel, C., Martz-Irngartinger, A., Linder, C., \& Schultze-Lutter, F. (2015). Age matters in the prevalence and clinical significance of ultra-high-risk for psychosis symptoms and criteria in the general population: Findings from the BEAR and BEARS-kid studies. World Psychiatry, 14(2), 189-197.

Schultze-Lutter, F., Debbane, M., Theodoridou, A., Wood, S. J., Raballo, A., Michel, C., et al. (2016). Revisiting the Basic Symptom Concept: Toward Translating Risk Symptoms for Psychosis into Neurobiological Targets. Front Psychiatry, 7, 9.

Schultze-Lutter, F., Michel, C., Schmidt, S. J., Schimmelmann, B. G., Maric, N. P., Salokangas, R. K., et al. (2015). EPA guidance on the early detection of clinical high risk states of psychoses. Eur Psychiatry, 30(3), 405-416.

Shamay-Tsoory, S. G., \& Aharon-Peretz, J. (2007). Dissociable prefrontal networks for cognitive and affective theory of mind: a lesion study. Neuropsychologia, 45(13), 3054-3067.

Solms, M. (2013). The conscious id. Psyche-Zeitschrift Fur Psychoanalyse Und Ihre Anwendungen, 67(9-10), 991-+.

van Donkersgoed, R. J. M., De Jong, S., Van der Gaag, M., Aleman, A., Lysaker, P. H., Wunderink, L., et al. (2014). A manual-based individual therapy to improve metacognition in schizophrenia: protocol of a multi-center RCT. BMC Psychiatry, 14. 
Figure 1: (a) The trans-theoretical model of developing psychotic disorders (adapted from Debbané, 2015)

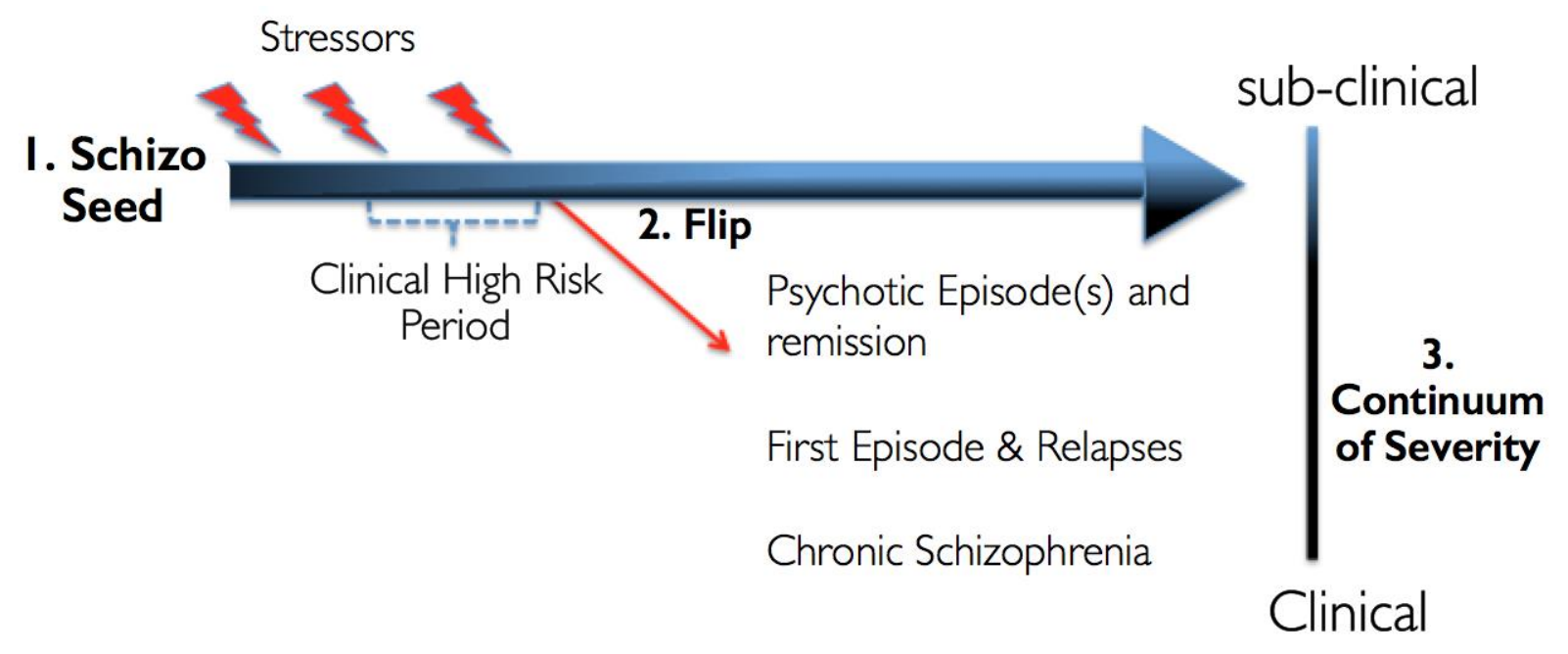

